\title{
Early exposure to germs and the Hygiene Hypothesis
}

\author{
Dale T Umetsu ${ }^{1}$ \\ IDivision of Immunology, Karp Laboratories, Rm 10127, Children's Hospital Boston, Harvard Medical School, One Blackfan Circle, \\ Boston, MA 02115, USA \\ Cell Research (2012) 22:1210-1211. doi:10.1038/cr.2012.65; published online 24 April 2012
}

\begin{abstract}
A recent paper suggests that reduced exposure to germs results in the expansion of a cell type called natural killer $T$ cells, which predisposes to colitis and asthma. Such a scenario could explain the Hygiene Hypothesis, which has been a puzzle for decades.
\end{abstract}

The Hygiene Hypothesis was proposed more than twenty years ago by Strachan [1] to explain the dramatic increase in the prevalence of allergic diseases and asthma that has occurred over the past two to three decades. What Strachan observed was that the younger children in large families had less asthma and allergy, presumably due to increased exposure to infections that passed around in such large families. Later, the Hygiene Hypothesis was extended to explain the great increase in the prevalence of inflammatory bowel disease that occurred over the same time period. However, the exact scientific underpinnings for the Hygiene Hypothesis, e.g., the specific infections and the mechanisms by which infections affect the immune system to prevent disease, have remained a puzzle over the years for both scientists and clinicians.

A clear biological explanation for the Hygiene Hypothesis though, may be at hand, as proposed in an article recently published in Science, using mouse mod-

Correspondence: Dale T Umetsu

E-mail: dale.umetsu@childrens.harvard.edu els [2]. Blumberg and his colleagues showed that mice raised under sterile, germ free conditions were more likely to develop experimental colitis, called oxazolone-induced colitis, and more likely to develop an experimental form of allergic asthma. Importantly, reestablishment of the intestinal commensal bacteria, collectively known as microbiota, in the germ-free mice with standard mouse colony bacteria prevented their predisposition to severe colitis or asthma. However, the beneficial effects from the microbiota developed only when very young mice were exposed to the bacteria, whereas exposure of adult germ-free mice to the microbiota did not reduce the predisposition to colitis or asthma. Thus, exposure of pregnant germ-free mice to the microbiota, which then affected the pups when they were born, prevented the later predisposition of the pups as adults to colitis and asthma, consistent with the idea that exposure of young children to germs prevents asthma and allergy.

Blumberg and colleagues went on to show that in the mice shielded from exposure to microbes, the predisposition to colitis and asthma was caused by an expansion of an inflammatory cell type called invariant natural killer $\mathrm{T}$ (iNKT) cells. Thus, in the colon and in the lungs of the germ-free mice, the investigators found a significant increase in the number of iNKT cells, which are required for the development of colitis and allergic asthma [3, 4]. Moreover, treatment of the germ-free mice with an anti-CD1d mAb, which prevented the activation of iNKT cells, also prevented the development of oxazolone-induced colitis and allergic asthma.

NKT cells comprise a fascinating subset of $\mathrm{T}$ cells that share characteristics with NK cells. NKT cells have been subdivided into two major groups, type I and type II. Type I NKT cells express a semi invariant (i) TCR and are known as invariant NKT (iNKT) cells, whereas type II NKT cells express a more diverse TCR repertoire. Both are activated by glycolipid antigens presented in the context of a class 1-like MHC molecule, called CD1d. Because the iTCR of iNKT cells is highly conserved in most mammals, and because iNKT cells with the iTCR are already widely expanded in vivo even in naïve mice, and are poised to rapidly respond and secrete large amounts of cytokines, including IFN- $\gamma$ and IL-4, iNKT cells are thought to play an important role in innate immunity. iNKT cells can respond to some bacteria by directly recognizing glycolipid antigens expressed by bacteria such as Sphingomonas, Helicobacter pylori, Streptococus pneumonea and Group B streptococcus, and can respond indirectly to many other bacteria such as Salmonella enterica and Staphylococcus aureas. Of note, glycolipids from H. pylori can expand a suppressor iNKT cell subset that can prevent the development of experimental asthma in mice [5]. Finally, iNKT cells can respond to 
glycolipid allergens present in pollens and in some foods $[6,7]$. However, the precise reasons why inflammatory iNKT cells expand in germ-free mice, or how exposure to environmental bacteria prevents the expansion of iNKT cells is not yet known.

While the role of iNKT cells in mouse models of colitis and asthma has been extensively studied, the role of iNKT cells in human disease is less well understood. In humans with ulcerative colitis, a form of inflammatory bowel disease, type II NKT cells producing large amounts of IL-13, appear to be associated with disease. In humans with asthma, iNKT cells have been found to be present in the lungs of some but not all individuals with asthma, leading to some controversy regarding the importance of iNKT cells in human asthma. However, it has become clear over the last few years from studies in humans and in mice that asthma is very heterogeneous, and that multiple pathways are involved in the development of asthma, each involving distinct cell types, including Th2 cells, iNKT cells, Th17 cells, nuocytes, Th9 cells and others. Finally, iNKT cells in humans have been shown to regulate transplantation tolerance, cancer and infection, suggesting that exposure to microbiota could have significant effects on a number of distinct human diseases.

The idea that mice growing up in "germless" environments develop an expansion of iNKT cells, which then predispose them to the development of colitis and allergic asthma, may be very relevant to humans. Epidemiological studies have already shown that birth by Caesarian section, which may limit early exposure to microbiota from the mothers' vaginal tracts, or extensive use of antibiotics in young children, which may also reduce the intestinal microbiota, also predisposes to the development of asthma and allergy. On the other hand, living on a farm in Western Europe, or having multiple pets, which may increase the early exposure to a more diverse community of microbiota, in some way prevents the development of asthma and allergy [8]. Thus, early events in young children related to microbial exposures appear to have lasting effects on the innate and adaptive immune systems, and as proposed by the Blumberg study, on iNKT cells.

The striking effects of commensal microbiota on the development iNKT cells are in line with other recent studies showing that specific strains of intestinal bacteria in mice, such as segmented filamentous bacteria, and clostridia are required for the development of Th17 cells [9] and regulatory $\mathrm{T}$ cells [10], respectively. Thus, it is becoming clear that a symbiotic relationship exists between bacteria and our immune system. As we understand this relationship better, perhaps in the future we could see the development of therapies that mimic the beneficial effects of "infection", for example, encouraging early exposure of infants to a "standard" disease-preventing collection of germs.

\section{References}

1 Strachan DP. Hay fever, hygiene, and household size. BMJ 1989; 299:12591260.

2 Olszak T, An D, Zeissig S, et al. Microbial exposure during early life has persistent effects on natural killer T cell function. Science 2012; 336:489-493.

3 Heller F, Fuss I, Nieuwenhuis E, Blumberg R, Strober W. Oxazolone colitis, a Th 2 colitis model resembling ulcerative colitis, is mediated by IL-13-producing NK-T cells. Immunity 2002; 17:629638.

4 Akbari O, Stock P, Meyer E, et al. Essential role of NKT cells producing IL-4 and IL-13 in the development of allergen-induced airway hyperreactivity. Nat Med 2003; 9:582-588.

5 Chang YJ, Kim HY, Albacker LA, et al. Influenza infection in suckling mice expands an NKT cell subset that protects against airway hyperreactivity. $J$ Clin Invest 2011; 121:57-69.

6 Agea E, Russano A, Bistoni O, et al. Human CD1-restricted T cell recognition of lipids from pollens. $J$ Exp Med 2005; 202:295-308.

7 Brennan PJ, Tatituri RV, Brigl M, et al. Invariant natural killer $\mathrm{T}$ cells recognize lipid self antigen induced by microbial danger signals. Nat Immunol 2011; 12:1202-1211.

8 Ege MJ, Mayer M, Normand AC, et al. Exposure to environmental microorganisms and childhood asthma. $N$ Engl J Med 2011; 364:701-709.

9 Ivanov II, Atarashi K, Manel N, et al. Induction of intestinal Th17 cells by segmented filamentous bacteria. Cell 2009; 139:485-498.

10 Atarashi K, Tanoue T, Shima T, et al. Induction of colonic regulatory $\mathrm{T}$ cells by indigenous Clostridium species. Science 2011; 331:337-341. 